\title{
CESTA BÁSICA NACIONAL: ANÁLISE NUTRICIONAL E FINANCEIRA
}

\section{NATIONAL FOOD BASKET: NUTRITIONAL ANALYSIS AND ECONOMIC}

\author{
Alana Binkoski ${ }^{1}$, Débora Fernandes Pinheiro ${ }^{2}$, Vania Schmitt ${ }^{3}$, Caryna Eurich Mazur ${ }^{4}$
}

1 - Nutricionista. Graduada pelo Centro Universitário Campo Real, Guarapuava - PR.

2 - Nutricionista. Mestranda em Produção Vegetal - Universidade Estadual do Centro Oeste (UNICENTRO)

- Guarapuava/PR, Brasil.

3 - Nutricionista. Docente do Centro Universitário Campo Real e Universidade Estadual do Centro Oeste. Doutoranda em Desenvolvimento Comunitário - Universidade Estadual do Centro Oeste (UNICENTRO) Guarapuava/PR, Brasil.

4 - Nutricionista. Doutora em Medicina Interna - Universidade Federal do Paraná (UFPR). Docente da Universidade Estadual do Centro Oeste (UNICENTRO) - Guarapuava/PR, Brasil.

*Autor para correspondência: deeh.fernandes@yahoo.com.br

\section{RESUMO:}

A cesta básica nacional é composta por treze itens básicos para a alimentação adequada de uma pessoa adulta. Assim, inúmeras pesquisas são realizadas com o intuito de verificar o preço da cesta básica, porém o cálculo nutricional bem como adequação de nutrientes não é verificado com tanta frequência. Portanto, o objetivo desse estudo foi verificar a média de preço e o valor nutricional da cesta básica nacional composta por treze itens prédeterminados em uma cidade do Paraná, Brasil. Para isso, foi realizada uma análise de prateleira e rótulo utilizando-se de tabelas nutricionais do Brasil nos seguintes alimentos: carne, leite, feijão, arroz, farinha, batata, tomate, pão francês, café em pó, banana, açúcar, óleo e manteiga. Bem como sua adequação de macronutrientes e micronutrientes para indivíduos adultos do gênero feminino e masculino de diferentes faixas etárias. Foram encontrados valores acima do recomendado para carboidratos (70,54\%), valores abaixo da recomendação para lipídeos $(15,32 \%)$ e adequação em relação à quantidade de proteínas $(14,12 \%)$. Os valores de vitamina A e de cálcio apresentaram déficit (27,54\% e 67,77\%, respectivamente). A cesta básica nacional indica deficiência de vitamina $A$ e cálcio, juntamente excedente de carboidratos. Contudo, o custo da cesta básica acaba sendo mais acessível às camadas mais populares. Todavia, é necessário que sejam realizadas adaptações para que ela atinja adequações nutricionais dentro da recomendação, a fim de promover alimentação adequada e saúde à população brasileira.

Palavras chave: alimentação, composição nutricional, macronutrientes, micronutrientes.

\section{ABSTRACT:}

The national food basket consists of thirteen basic food items suitable for an adult. Numerous researches are carried out in order to check the price of the basket. But the nutritional calculation as well as appropriateness of nutrients are not checked as often. To verify the average price and the nutritional value of the food basket composed of thirteen national predetermined items in a city of Paraná, Brazil. Analysis and shelf label using nutritional tables of Brazil in the following foods: meat, milk, beans, rice, flour, potatoes, tomatoes, French bread, coffee, bananas, sugar, oil and butter. As well as appropriateness of your macronutrients and micronutrients for adults of the female gender and different age groups male. Were found above the recommended values for carbohydrates $(70.54 \%)$, values below the recommendation for lipids (15.32\%) and appropriateness in relation to the amount of protein (14.12\%). The values of vitamin A and calcium showed a deficit (27.54\% 
e $67.77 \%$, respectively). The national basket indicates deficiency of vitamin A and calcium, along surplus of carbohydrates. However, the cost of the basic basket ends up being more accessible to most popular layers. However, they must be carried out adaptations so that it reaches nutritional adequacies in the recommendation, in order to promote proper nutrition and health to the brazilian population.

Keywords: alimentation, nutritional composition, macronutrients, micronutrients.

\section{INTRODUÇÃO}

A ingestão de nutrientes propiciada pela alimentação é essencial para a manutenção da saúde da população (BRASIL, 2014). Contudo, o Brasil passou por uma transição nutricional ocorrida neste século, resultado da globalização da dieta, caracterizada pelos altos teores de gorduras, principalmente de origem animal, de açúcares e alimentos refinados e baixos teores de carboidratos complexos e fibras (MONTEIRO et al., 2000). Dados da Pesquisa de Orçamento Familiar (POF) de 2011 confirmam que as mudanças de padrão alimentar no país, de modo geral, são desfavoráveis no que se refere às doenças carenciais, hipovitaminose $\mathrm{A}$ e as demais doenças crônicas não transmissíveis (DCNT) devido ao aumento no consumo de gorduras e diminuição com relação aos vegetais, frutas e hortaliças (POF, 2011).

Assim, em relação à alimentação do brasileiro, segundo o Decreto Lei nํㅜ 399 de 1938 foi implantada a chamada "Ração Essencial Mínima", popularmente conhecida como cesta básica nacional, na qual foram determinados 13 itens alimentícios que tem como objetivo ser suficiente para garantir, durante um mês, o sustento e bem-estar de um trabalhador em idade adulta, contendo quantidades balanceadas de calorias, proteínas, cálcio e ferro (BRASIL, 1938). Sendo composta por treze itens alimentares: carne, leite, feijão, arroz, farinha, batata, legumes (tomate), pão francês, café em pó, frutas (banana), açúcar, óleo e manteiga (DIEESE, 2009).

Sabe-se que, atualmente o Departamento Intersindical de Estatística e Estudos Socioeconômicos (DIEESE) realiza mensalmente em 16 capitais brasileiras uma pesquisa referente ao custo que os 13 itens alimentares da Cesta Básica Nacional têm para os trabalhadores, e compara os dados com o valor do salário mínimo vigente (PASSOS; BERNARDI; MENDES, 2014). Somente o custo da Cesta Básica Nacional é calculado, não existindo dados referentes ao seu valor nutricional, quanto a macronutrientes e micronutrientes (DIEESE, 2009).

De tal modo, o acompanhamento dos preços das cestas básicas no Brasil segue critérios rigorosos e sistemáticos, tornando-se um importante indexador para salários e a 
evolução do poder de compra nos estratos de baixa renda (AMORIM; SOUSA; CORONEL, 2013).

Portanto, o custo da cesta básica é um dos instrumentos importantes para a avaliação do desenvolvimento socioeconômico e nutricional de uma região e a relevância da análise da integração de preços é uma forma de aumentar a eficiência do mercado (AMORIM; SOUSA; CORONEL, 2013).

Em 2016, o valor acumulado da cesta básica aumentou nas 27 capitais do país, onde a DIEESE realiza mensalmente, durante todo o ano, a Pesquisa Nacional da Cesta Básica de Alimentos (DIEESE, 2017).

Deste modo, o objetivo do presente estudo é analisar o valor médio da Cesta Básica Nacional no Município de Guarapuava-Paraná, bem como descrever o valor nutricional (energia, macro e micronutrientes) fornecido pelos 13 itens da cesta, verificando se esses são adequados para as recomendações de uma pessoa adulta.

\section{MATERIAL E MÉTODOS}

\subsection{Caracterização do estudo}

Trata-se de um estudo transversal com caráter quantitativo. Foram avaliados os itens componentes da cesta básica para verificar o custo médio mensal da cesta para um adulto no município de Guarapuava-Paraná. Também, foram avaliados os valores nutricionais diários fornecidos pelo consumo da cesta comparados às suas necessidades nutricionais, descritas na Dietary References Intakes (DRI, 2010).

\subsection{Coleta de dados}

A coleta de dados foi realizada em 4 supermercados diferentes no município de Guarapuava-PR no período de outubro de 2017, onde foram feitos os levantamentos de preços e rótulos nutricionais dos 13 itens da Cesta Básica (descritos no DIEESE). Para determinação dos alimentos a serem analisados, foram escolhidos os que apresentaram menor valor aquisitivo, devido principalmente ao acesso pela população de menor poder aquisitivo.

Os supermercados foram escolhidos por conveniência, localizados em regiões periféricas, em bairros com características residenciais. Para a realização da pesquisa de 
preços nos supermercados, foi empregado um termo de consentimento e aprovação da coleta de dados assinada pelos responsáveis do estabelecimento, onde estava claro que a pesquisa realizada não implicaria em nenhum dano e/ou prejuízo à empresa, não havendo publicação de valores e nomes de quaisquer pessoas ou pontos comerciais. Todos os supermercados afirmaram estar de acordo com a pesquisa realizada (Anexo 1).

\subsection{Caracterização e análise da amostra}

Segundo dados da DIEESE, é possível obter valores referentes às quantidades per capitas mensais de cada item da cesta. A partir desses dados, foi possível determinar as quantidades de per capitas diários, estimados para cada alimento.

TABELA 1. Per capitas mensais dos itens da cesta básica nacional, Guarapuava/PR, Brasil.

\begin{tabular}{cc}
\hline Alimento & Per capitas diários \\
\hline Carne (filé agulha) & $220 \mathrm{~g}$ \\
Leite & $250 \mathrm{ml}$ \\
Feijão & $150 \mathrm{~g}$ \\
Arroz & $100 \mathrm{~g}$ \\
Farinha de trigo & $50 \mathrm{~g}$ \\
Batata & $200 \mathrm{~g}$ \\
Legumes (tomate) & $300 \mathrm{~g}$ \\
Pão caseiro & $200 \mathrm{~g}$ \\
Café em pó & $20 \mathrm{~g}$ \\
Frutas (banana) & $600 \mathrm{~g}$ \\
Açúcar cristal & $100 \mathrm{~g}$ \\
Óleo de soja & $30 \mathrm{ml}$ \\
Manteiga & $25 \mathrm{~g}$ \\
\hline
\end{tabular}

Fonte: Binkoski et al., (2017). 
Os dados obtidos através da pesquisa nos supermercados, foram tabulados em planilha própria dos preços da cesta básica e feita a análise descritiva dos valores dos produtos e valores totais de cada supermercado. Em seguida foi realizada a média dos preços da cesta básica dos 4 supermercados, considerando o percentual comprometido do salário mínimo vigente ( $\mathrm{R} \$ 937,00$ reais) para a compra dos itens.

Foram realizados cálculos de avaliação e adequação nutricional, comparadas às recomendações da DRI, por meio da Recommended Dietary Allowances (RDA) quanto ao fornecimento de energia e nutrientes para um indivíduo adulto saudável. $A$ análise das recomendações diárias foi separada por gênero e por idade no caso da mulher (Homem de 19 a 70 anos, mulher de 19 a 50 anos e mulher de 51 a 70 anos), devido a algumas diferenças nas recomendações entre as faixas etárias, e também às mulheres serem os maiores percentuais em relação à chefia da família.

TABELA 2. Valores diários recomendados de nutrientes.

\begin{tabular}{|c|c|c|c|}
\hline Nutrientes & $\begin{array}{l}\text { Gênero Masculino } \\
\text { (19 - } 70 \text { anos) }\end{array}$ & $\begin{array}{c}\text { Gênero Feminino } \\
\text { (19 - } 50 \text { anos) }\end{array}$ & $\begin{array}{c}\text { Gênero Feminino } \\
\text { (51 - } 70 \text { anos) }\end{array}$ \\
\hline & RDA & RDA & RDA \\
\hline $\mathrm{CHO}(\%)$ & 45 a 65 & 45 a 65 & 45 a 65 \\
\hline PTN (\%) & 10 a 35 & 10 a 35 & 10 a 35 \\
\hline LIP (\%) & 25 a 35 & 25 a 35 & 25 a 35 \\
\hline $\mathrm{Ca}(\mathrm{mg})$ & 1000 & 1000 & 1200 \\
\hline $\mathrm{Fe}(\mathrm{mg})$ & 8 & 18 & 8 \\
\hline$P(\mathbf{m g})$ & 700 & 700 & 700 \\
\hline $\mathrm{Zn}(\mathrm{mg})$ & 11 & 8 & 8 \\
\hline Vit. A ( $\mu \mathrm{g})$ & 900 & 700 & 700 \\
\hline Vit. C (mg) & 90 & 75 & 75 \\
\hline
\end{tabular}

mg - miligramas; $\mu \mathrm{g}$ - microgramas; DP - desvio padrão.

Fonte: Binkoski et al., (2017).

De acordo com o preconizado pela DRI (2010), o cálcio tem como recomendação 1000 mg para o gênero masculino (19-70 anos) e gênero feminino (19-50 anos) e 1200 mg para o gênero feminino (51-70 anos). A recomendação de ferro é de $8 \mathrm{mg}$ para o gênero masculino e feminino (51-70) e $18 \mathrm{mg}$ para o gênero feminino (19-50). O fósforo tem como 
recomendação $700 \mathrm{mg}$ para ambos os gêneros. A quantidade de zinco recomendada é 11 mg para o gênero masculino e $8 \mathrm{mg}$ para ambas as faixas etárias do gênero feminino. Os valores de vitamina A recomendados pela RDA são $900 \mu \mathrm{g}$ para o gênero masculino e 700 $\mu \mathrm{g}$ para o gênero feminino. $\mathrm{O}$ consumo de vitamina $\mathrm{C}$ é preconizado em $90 \mathrm{mg}$ para $\mathrm{O}$ gênero masculino e 75 mg para o gênero feminino.

Foi realizada a análise nutricional dos per capitas diários dos alimentos componentes da cesta. Para a contagem de macronutrientes (carboidratos, proteínas e lipídeos), foram utilizados os rótulos nutricionais dos itens industrializados e a Tabela Brasileira de Composição de Alimentos para os alimentos in natura. Para os cálculos de micronutrientes (cálcio, ferro, fósforo, zinco, vitamina A e vitamina C), a tabela TACO foi utilizada para todos os alimentos.

Para obter-se o valor energético total da cesta, foram utilizados os valores de macronutrientes encontrados, multiplicando os valores de carboidratos e proteínas (em gramas) por 4 kcal/grama e o valor de lipídeos (em gramas) por 9 kcal/gramas.

\subsection{Análise estatística}

Os resultados foram avaliados por meio de estatística descritiva no software Microsoft Excel® versão 2017 para Windows.

\section{RESULTADOS E DISCUSSÃO}

A Tabela 3 mostra os valores da Cesta Básica dos 4 supermercados de Guarapuava-PR, onde foi realizada a pesquisa de preços, bem como a média entre os 4 diferentes valores encontrados e a porcentagem do Salário Mínimo a ser comprometida com a compra do item.

O Supermercado 1 teve o valor da Cesta Básica determinado de $R \$ 203,94 \pm 18,95$ reais, compreendendo $21,77 \%$ do Salário Mínimo. O supermercado 2 teve o custo da cesta $\mathrm{R} \$ 184,48 \pm 11,65$ reais, representando $19,69 \%$ do salário. O supermercado 3 obteve o valor da cesta calculado em $R \$ 188,50 \pm 14,47$ reais, tendo seu custo $R \$ 4,02$ reais maiores em comparação ao supermercado 2, apresentando percentual de comprometimento do salário de $21,84 \%$. O supermercado 4 apresenta o maior valor encontrado, sendo R $\$ 242,64$ $\pm 19,13$ reais, ou seja, o custo da cesta é quase $24 \%$ maior em relação ao preço encontrado no supermercado 2, o qual apresentou o menor custo da cesta. 
Entre os 4 supermercados analisados, o custo médio que um trabalhador adulto possui para a aquisição da Cesta Básica foi de $R \$ 204,89 \pm 26,52$ reais, dispondo de $22,30 \%$ do salário mínimo vigente que é de $R \$ 937,00$ reais.

Tabela 3. Valor médio em reais da Cesta Básica Nacional em relação ao equivalente do salário mínimo brasileiro, município de Guarapuava-PR.

Valor da Cesta Média DP

\begin{tabular}{ccc}
\hline Supermercado 1 & $\mathrm{R} \$ 203,94 \pm 18,95$ & $21,77 \%$ \\
Supermercado 2 & $\mathrm{R} \$ 184,48 \pm 11,65$ & $19,69 \%$ \\
Supermercado 3 & $\mathrm{R} \$ 188,50 \pm 14,47$ & $21,87 \%$ \\
Supermercado 4 & $\mathrm{R} \$ 242,64 \pm 19,13$ & $25,9 \%$ \\
Média & $\mathrm{R} \$ 204,89 \pm 26,52$ & $22,30 \%$ \\
\hline
\end{tabular}

Fonte: Binskoski et al., (2017).

A Tabela 4 apresenta dados referentes aos valores de carboidratos, proteínas, lipídeos, cálcio, ferro, fósforo, vitamina A e vitamina C, fornecidos pela Cesta Básica, contrapostos com os valores sugeridos pela RDA (DRI, 2010).

Em relação aos macronutrientes, a média das Cestas Básicas encontradas é composta por $70,54 \%$ de carboidratos, $14,12 \%$ de proteínas e $15,32 \%$ de lipídeos. Segundo estes valores, verificaram-se adequados segundo a RDA apenas para proteínas. Os carboidratos apresentaram-se 5,54\% acima do percentil máximo recomendado pela RDA (45 a 65\%). Em contrapartida, o lipídeo comportou-se 9,68\% abaixo do limite mínimo preconizado pela RDA (25 a 35\%).

A análise de micronutrientes resultou em valores adequados para o consumo de ferro, fósforo, zinco e vitamina C para ambos os sexos. Todos os micronutrientes no presente trabalho obtiveram desvio padrão iguais à zero, devido à análise nutricional dos mesmos dar-se pela tabela TACO e então serem padronizados igualmente.

Com isso, do Cálcio representado na Cesta Básica é 869,45 mg, apresentando-se 13,05\% abaixo das necessidades de $1000 \mathrm{mg}$ para o gênero masculino (19-70 anos) e gênero feminino (19-50 anos), bem como 27,54\% abaixo das necessidades para o gênero feminino (51-70 anos). Para vitamina A, o valor encontrado na cesta é $290 \mu \mathrm{g}$, estando 
$67,77 \%$ abaixo dos valores recomendados para o gênero masculino e 58,57\% para o gênero feminino.

A média do valor calórico total fornecido pelas cestas analisadas é de 3864,68 kcal/dia. Segundo a recomendação do Guia Alimentar para a População Brasileira (2006), a ingestão calórica média recomendada para adultos é com base em uma dieta de 2000 kcal. Deste modo, a Cesta Básica fornece $48,24 \%$ de calorias a mais do que valor médio recomendado.

Tabela 4. Valores de nutrientes encontrados na Cesta Básica Nacional do Munícipio de Guarapuava bem como suas recomendações diárias de nutrientes.

\begin{tabular}{|c|c|c|c|c|c|c|}
\hline \multirow{3}{*}{ Nutrientes } & \multicolumn{2}{|c|}{$\begin{array}{c}\text { Gênero Masculino } \\
\text { (19 - } 70 \text { anos) }\end{array}$} & \multicolumn{2}{|c|}{$\begin{array}{c}\text { Gênero Feminino } \\
\text { (19 - 50 anos) }\end{array}$} & \multicolumn{2}{|c|}{$\begin{array}{c}\text { Gênero Feminino } \\
\text { (51 - } 70 \text { anos) }\end{array}$} \\
\hline & & Média & & Média & & Média \\
\hline & RDA & Cesta \pm DP & RDA & $\begin{array}{c}\text { Cesta } \pm \\
\text { DP }\end{array}$ & RDA & $\begin{array}{c}\text { Cesta } \pm \\
\text { DP }\end{array}$ \\
\hline CHO (\%) & 45 a 65 & $70,54 \pm 3,80$ & \multirow{2}{*}{$\begin{array}{l}45 a \\
65 \%\end{array}$} & \multirow{2}{*}{$\begin{array}{c}70,54 \% \pm \\
3,80\end{array}$} & \multirow{2}{*}{$\begin{array}{c}45 a \\
65\end{array}$} & \multirow{2}{*}{$\begin{array}{c}70,54 \% \pm \\
3,80\end{array}$} \\
\hline PTN (\%) & 10 a 35 & $14,12 \pm 0,59$ & & & & \\
\hline LIP (\%) & 25 a 35 & $15,32 \pm 4,10$ & $\begin{array}{l}10 a \\
35 \%\end{array}$ & $\begin{array}{c}14,12 \% \pm \\
0,59\end{array}$ & $\begin{array}{c}10 a \\
35\end{array}$ & $\begin{array}{c}14,12 \% \pm \\
0,59\end{array}$ \\
\hline $\mathrm{Ca}(\mathrm{mg})$ & 1000 & $869,45 \pm 0$ & $25 a$ & $15,32 \% \pm$ & $25 a$ & $15,32 \% \pm$ \\
\hline $\mathrm{Fe}(\mathrm{mg})$ & 8 & $29,32 \pm 0$ & $35 \%$ & 4,10 & 35 & 4,10 \\
\hline$P(\mathbf{m g})$ & 700 & $1946,6 \pm 0$ & 1000 & $869,45 \pm 0$ & 1200 & $869,45 \pm 0$ \\
\hline Zn (mg) & 11 & $21,13 \pm 0$ & 18 & $29,3 \pm 0$ & 8 & $29,32 \pm 0$ \\
\hline Vit. A ( $\mu \mathrm{g})$ & 900 & $290,1 \pm 0$ & 700 & $1946,6 \pm 0$ & 700 & $1946,6 \pm 0$ \\
\hline \multirow[t]{3}{*}{ Vit. C (mg) } & 90 & $220 \pm 0$ & 8 & $21,13 \pm 0$ & 8 & $21,13 \pm 0$ \\
\hline & & & 700 & $290,1 \pm 0$ & 700 & $290,1 \pm 0$ \\
\hline & & & 75 & $220 \pm 0$ & 75 & $220 \pm 0$ \\
\hline
\end{tabular}

mg - miligramas; $\mu g$ - microgramas; DP - desvio padrão.

Fonte: Binkoski et al., (2017).

Em um estudo publicado em 2017, o preço médio da cesta básica na cidade de Curitiba, Paraná foi de $R \$ 409,86$ reais e na capital de São Paulo o valor médio foi de $R \$$ 438,89 reais (DIESSE, 2017). No presente estudo a média de preço apresentou-se em $R \$$ 204,89 reais. Essa discrepância pode ser explicada, pois estudos nos trazem que o custo de vida é maior em regiões metropolitanas no Brasil (ALMEIDA; AZZONI, 2016). Todas as 
pesquisas de preço realizadas pela DIEESE são em regiões metropolitanas, ainda não há um comparativo em relação a regiões do interior dos estados brasileiros.

Em contrapartida, uma pesquisa de preços realizada pelo PROCON no ano de 2016, na cidade de Joinville, estado de Santa Catarina, analisou um menor preço da cesta básica, de $\mathrm{R} \$ 197,44$ e o maior de $\mathrm{R} \$ 289,19$ (PROCON, 2016). Da mesma forma ocorreu variação de valores muito semelhantes no presente estudo, com o menor preço da cesta básica no valor de $R \$ 184,48$ reais e o maior de $R \$ 242,64$ reais. Confirmando isto, Amorim et al. (2013), diz que não é presente a Lei do Preço Único nos mercados em relação a compra das cestas básicas.

Em relação ao percentual utilizado do salário mínimo, percebe-se discrepância dos valores quando comparados ao preço das cestas nas regiões metropolitanas, sendo em média de 48,9\% (DIEESE, 2017), com discrepância de 26,6\% do presente estudo. Nesse estudo foi observada a média de $22,3 \%$ do salário mínimo do corrente ano. Importante ressaltar que, durante o período de 2003 a 2012 o salário mínimo nacional cresceu 70\% (SALTIEL; URZÚA, 2017). Engbom e Moser (2017) mostraram que o salário mínimo resultou em aumentos significativos nos ganhos de salários dos trabalhadores e que de fato, desempenha um papel importante na redução da desigualdade social.

Devido ao valor da cesta básica comprometer significativamente a renda pode ocorrer ferimento à Segurança Alimentar e Nutricional, a qual refere-se à forma como uma sociedade organizada (por meio de políticas públicas, da responsabilidade do Estado e da sociedade em geral) pode e deve garantir o Direito Humano à Alimentação Adequada (DHAA) a todos os cidadãos, oque permite o alcance de forma digna do estado de segurança alimentar e nutricional da população (ABRANDH, 2013). Entretanto, para associar os dados de valores das cestas básicas e insegurança alimentar é preciso a condução de outros estudos, com uma população maior.

No estudo de Passos et al. (2013), foram analisados os valores de nutrientes encontrados na cesta básica nacional e pode-se perceber que, a quantidade de lipídeo estava muito acima do adequado, com $44,42 \%$ do valor total. Já no presente trabalho a quantidade de lipídeos apresentados foi de 14,12\%, estando abaixo do recomendado.

Dessa forma, se faz necessário aumentar a oferta de lipídeos para tornar o valor compatível com as recomendações dietéticas. O aumento de óleos vegetais seria uma das soluções para o aumento desse macronutriente. Porém, é preciso atentar para que não ocorra aumento superior às necessidades, visto que a alta concentração de lipídeos circulantes sanguíneos é um fator de risco para o desenvolvimento da doença 
aterosclerótica (ANDERSON et al., 2014), doença hepática gordurosa não alcoólica (PINKOSKY et al., 2017), além de uma alta taxa de mortalidade (COFFEY et al., 2017).

Apesar de serem cada vez mais observadas doenças cardiovasculares, uma dieta com inadequação de lipídeos é maléfica para a saúde. Pois, dos pontos de vista fisiológico e clínico, os lipídeos biologicamente mais relevantes são os fosfolipídeos, o colesterol, os triglicerídeos (TG) e os ácidos graxos. Os fosfolipídeos formam a estrutura básica das membranas celulares, o colesterol é precursor dos hormônios esteroides, dos ácidos biliares e da vitamina $D$, além disso, como constituinte das membranas celulares, 0 colesterol atua na fluidez destas e na ativação de enzimas aí situadas, e os TG constituem uma das formas de armazenamento energético mais importante no organismo, sendo depositados nos tecidos adiposo e muscular (FALUDI et al., 2017). Desta forma, podem haver prejuízos quando a dieta for hipolipídica como demonstrado na análise da cesta básica.

Além de trabalhos revelarem que níveis mais baixos de colesterol estavam associados a ingestões mais baixas de gorduras saturadas, e de que maior ingestão de gordura poli-insaturada e aumento dos níveis de colesterol HDL estavam relacionados a maiores quantidades de gordura total (SCHWINGSHACKL e HOFFMANN, 2013). Dietas com baixa quantidade de gordura melhoram principalmente a perda de peso corporal (BAZZANO et al., 2014), além de melhorar o perfil lipídico do indivíduo (SCHWINGSHACKL e HOFFMAN, 2013).

Uma meta-análise demonstrou que as dietas com baixo teor de gordura e baixo teor de carboidratos, reduziram os níveis de colesterol LDL, embora a redução fosse menor para as pessoas atribuídas a dietas com baixo teor de carboidratos (HU et al., 2012).

Todavia os valores de carboidratos no presente estudo estão acima da média, sendo $70,54 \%$, com 5,54\% acima do preconizado, possuindo caráter de dieta hiperglicídica. Evidências crescentes ressaltam o importante papel do controle glicêmico na saúde e prevenção de doenças. Pesquisadores da Sociedade Europeia de Nutrição Enteral e Parental (ESPEN), puderam concluir que o excesso de disponibilidade de carboidratos principalmente glicose e frutose, podem resultar em impacto negativo na saúde (BARAZZONI et al., 2017). Além de seu aumento ocasionar vários problemas de saúde, contribuindo para o desenvolvimento da obesidade, resistência à insulina e diabetes mellitus tipo 2 (AL-GOBLAN; AL-ALFI; KHAN, 2014). Alimentos com alto índice glicêmico e carga glicêmica estão associadas com maior risco de tais doenças (BHUPATHIRAJU et al., 
2014), a inflamação pode ser diminuída pela modificação da dieta (ADAMSSON et al., 2015).

A deficiência de vitaminas afeta negativamente a imunidade do indivíduo (SALVA et al., 2012; MORA; IWATA; VON ANDRIAN, 2010). Para que o sistema imunológico funcione eficientemente, o bom estado nutricional é imprescindível (EL-ZAYAT, 2017). Em relação a análise dos micronutrientes observa-se déficit nos níveis de vitamina $A$ e cálcio. Sendo este, o mineral mais abundante do corpo humano, envolvido em muitos processos fisiológicos e patológicos (PU; CHEN; XUE, 2016). Através da interação com numerosas proteínas distribuídas em diferentes compartimentos celulares, está envolto em uma grande quantidade de aspectos fisiológicos tais como a contração muscular, ativação enzimática, diferenciação celular, resposta imune, morte celular programada e atividade neuronal (ZHOU; XUE; YANG, 2013).

Os alimentos ricos em cálcio incluem leite, iogurte, queijo, camarão, soja, tofu, leite de soja, brócolis, laranja, couve e outros (PU; CHEN; XUE, 2016). A ingestão insuficiente resulta em doenças como osteoporose e raquitismo (UDAY; HOGLER, 2017), além de estarem mais propícios a desenvolver doenças neurológicas como Parkinson (GAO et al., 2015), também de aumentar o risco de fraturas, diminuir a densidade óssea (VANNUCl et al., 2017).

A deficiência de vitamina $A$ ocorre quando a ingestão de frutas e verduras é prejudicada, ou seja, de carotenoides de frutas e vegetais ou até mesmo a vitamina A préformada de produtos animais e lácteos (SOMMER, 2008), afetando principalmente crianças, gestantes e lactantes (WEDNER; ROSS, 2017). Em um estudo conduzido no Brasil com 1436 crianças, a prevalência do consumo inadequado de vitamina $A$ foi observada em 16,1\% da amostra (KONSTANTYNER; WARKENTIN; TADDEI, 2014). Esta vitamina é um nutriente essencial que desempenha um papel crucial na visão, diferenciação celular, desenvolvimento embrionário, resposta imune e desenvolvimento sistema nervoso (WEDNER; ROSS, 2017; BLOMHOFF; BLOMHOFF, 2006; BALMER; BLOMHOFF, 2002).

As carências de micronutrientes (vitamina A e cálcio) presentes na cesta básica podem ter se dado pela composição da mesma, visto que há poucos alimentos in natura, os quais são a maior fonte de fornecimento de vitaminas e minerais.

Sobretudo, podemos observar que a cesta básica média encontrada na cidade de Guarapuava, apesar de possuir inadequações em alguns nutrientes, em partes pode conter algumas adequações nutricionais para suprir as necessidades da população. Porém, deve haver atenção à ingestão de populações com alguma enfermidade que impeça o consumo 
de uma dieta hiperglícida, tal como diabetes, assim como para o consumo calórico excessivo.

\section{CONCLUSÃO}

Com o presente estudo pode-se observar o custo da cesta básica nacional na cidade de Guarapuava, a qual se apresentou com valores menores do que em outras regiões metropolitanas do Brasil. Isso é benéfico para a população, sendo mais acessível às camadas mais populares, fator positivo devido aos trabalhadores assalariados serem os maiores usuários deste tipo de produto.

No entanto, nesse estudo a cesta básica nacional indica deficiência de vitamina A e cálcio, juntamente com valores excedentes em carboidratos e calorias. Devido a isso, se fazem necessárias adaptações para que esta venha a ter melhor aporte nutricional. A fim de promover bem-estar e saúde à população brasileira. Apesar destas inadequações ela pode ser considerada como nutricionalmente saudável aos seus comensais.

\section{REFERÊNCIAS}

ABRANDH. O direito humano à alimentação adequada e o sistema nacional de segurança alimentar e nutricional. Marília Leão. Brasília, 2013.

ADAMSSON, V.; REUMARK, A.; MARKLUND, M.; LARSSON, A.; RISERUS, U. Role of a prudent breakfast in improving cardiometabolic risk factors in subjects with hypercholesterolemia: a randomized controlled trial. Clinical Nutrition, v.34, n.1, p.20-26, 2015.

AL-GOBLAN, A.S.; AL-ALFI, M.A.; KHAN, M.Z. Mechanism linking diabetes mellitus and obesity. Diabetes, Metabolic Syndrome and Obesity: Targets and Therapy, v.7, p.587-591, 2014.

ALMEIDA, A.N.; AZZONI, C.R. Custo de vida comparativo das Regiões Metropolitanas Brasileiras: 1996-2014. Estudos Econômicos (São Paulo), v.46, n.1, p.253-276, 2016.

AMORIM, A.L.; SOUSA, E.P.; CORONEL, D.A. Preço da cesta básica na Região Sul do Brasil: testando a integração espacial. Revista de Economia e Administração, v.12, n.3, p.321-348, 2013. 
ANDERSON, T.J.; MANCINI, G.B.; GENEST, J.JR.; GRÉGOIRE, J.; LONN, E.M.; HEGELE, R.A. The new dyslipidemia guidelines: what is the debate? Canadian Journal of Cardiology, v.31, n.5, p.605-612, 2014.

BAIN, L. E.; AWAH, P. K.; GERALDINE, N.; KINDONG, N. P.; SIGAL, Y.; BERNARD, N.; TANJEKO, A. T. Malnutrition in Sub-Saharan Africa: burden, causes and prospects. The Pan African Medical Journal, v. 15. P.120, 2013.

BALMER, J.E.; BLOMHOFF, R. Gene expression regulation by retinoic acid. Journal of lipid research, v.43, p.1773-1808, 2002

BARAZZONI, R.; DEUTZ, N.E.P.; BIOLO, G.; BISCHOFF, S.; BOIRIE, Y.; CEDERHOLM, T. et al. Carbohydrates and insulin resistance in clinical nutrition: Recommendations from the ESPEN expert group. Clinical Nutrition, v.36, n.2, p.355-363, 2017.

BAZZANO, L.A.; REYNOLDS, K.; YAO, L.; BRUNOL, C.; LIU, Y.; CHEN, C.S; KLAG, M.J. et al. Effects of low-carbohydrate and low-fat diets: a randomized trial. Annals of Internal Medicine, v.161, n.5, p.309-318, 2014.

BERGER, S.; RAMAN, G.; VISHWANATHAN, R.; JACQUES, P.F.; JOHNSON, E.J. Dietary cholesterol and cardiovascular disease: a systematic review and meta-analysis. The American Journal of Clinical Nutrition, v.102, n.2, p.276-94, 2015.

BHUPATHIRAJU, S.N.; TOBIAS, D.K.; MALIK, V.S.; PAN, A.; HRUBY, A.; MANSON, J.E. et al. Glycemic index, glycemic load, and risk of type 2 diabetes: results from 3 large US cohorts and an updated meta-analysis. The American Journal of Clinical Nutrition, v.100, n.1, p.218-232, 2014.

BLOMHOFF, R.; BLOMHOFF, H.K. Overview of retinoid metabolism and function. Journal of neurobiology, v.66, p.606-630, 2006.

BRASIL. Decreto Lei $n^{\circ}$ 399, de 30 de outubro de 1938. Diário Oficial da República Federativa do Brasil. Poder Executivo, Brasília, DF, 30 out. 1938. 
BRASIL. Ministério Da Saúde. Secretaria De Atenção À Saúde. Departamento De Atenção Básica. Guia alimentar para a população brasileira / ministério da saúde, secretaria de atenção à saúde, departamento de atenção Básica. - 2. ed. - Brasília: ministério da saúde, 156p., 2014.

CARMELIET, G.; DERMAUW, V.; BOUILLON, R. Vitamin D signaling in calcium and bone homeostasis: a delicate balance. Best Practice \& Research Clinical Endocrinology \& Metabolism, v.29, n.4, p. 621-631, 2015.

CHRISTENSE, J.J.; ULVEN, S.M.; RETTERSTOL, K.; NARVERUD, I.; BOGSRUD, M.P.; HENRIKSEN, T.; BOLLERSLEV, J. et al. Comprehensive lipid and metabolite profiling of children with and without familial hypercholesterolemia: A cross-sectional study.

COFFEY, A.R.; SMALLWOOD, T.L.; ALBRIGHT J.; HUA, K.; KANKE, M.; POMP, D.; BENNETT, B.J.; SETHUPATHY, P. Systems genetics identifies a co-regulated module of liver microRNAs associated with plasma LDL cholesterol in murine diet-induced dyslipidemia. Physiological Genomics, v.49, n.11, p.618-629, 2017.

DIEESE. Departamento Intersindical de Estatística e Estudos Socioeconômicos. Análise cesta básica 2016. São Paulo: 12p, 2017.

DIEESE. Departamento Intersindical de Estatística e Estudos Socioeconômicos. Metodologia da Cesta Básica de Alimentos. São Paulo: 24.p, 2009.

EL-ZAYAT, S.R.; SIBAII, H.; MAHFOUZ, N.N.; SALLAM, S.F.; FAHMY, R.F.; ELSHAHEED, A.A. Effects of vitamin A deficiency on thymosin- $\beta 4$ and CD4 concentrations. Journal of Genetic Engineering and Biotechnology, 2017.

ENGBOM, N.; MOSER, C. Earnings Inequality and the Minimum Wage: Evidence from Brazil. Princeton University. Working Paper, n.6393, 63p., 2017.

FALUDI, A.A.; IZAR, M.C.O.; SARAIVA, J.F.K.; CHACRA, A.P.M.; BIANCO, H.T.; AFIUNE NETO, A. et al. Atualização da Diretriz Brasileira de Dislipidemias e Prevenção da Aterosclerose. Arquivos Brasileiros de Cardiologia, v.109, n.1, p.1-76, 2017. 
GAO, H.; WEI, X.; LIAO, J.; WANG, R.; XU, J.; LIU, X.; PAN, X.; LI, Z.; LI, Z.; XIA, Y.; WANG, $Q$. Lower bone mineral density in patients with parkinson's disease: a cross-sectional study from Chinese Mainland. Frontiers in Aging Neuroscience, v.7, p.203, 2015.

HU, T.; MILLS, K.T.; YAO, L.; DEMANELIS, K.; ELOUSTAZ, M. et al. Effects of lowcarbohydrate diets versus low-fat diets on metabolic risk factors: a meta-analysis of randomized controlled clinical trials. American Journal of Epidemiology, v.176, n.7, p.44$54,2012$.

KONSTANTYNER, T.; WARKENTIN, S.; TADDEI, J.A.A.C. Prevalence and determinants of vitamin A deficiency among Brazilian children under 2 years of age from the 2006 National Demographic Health Survey. Food and Nutrition Bulletin, v.35, n.4, p.422-430, 2014.

MONTEIRO, C. A.; MONDINI, L.; COSTA R. B. L. Mudanças na composição e adequação nutricional da dieta alimentar nas áreas metropolitanas do Brasil (1988-1996). Revista de Saúde Pública, v. 34, n. 3, p.251-58, 2000.

MORA, J.M.; IWATA, M.; VON ANDRIAN, U.H. Vitamin effects on the immune system: vitamins $A$ and $D$ take center stage. Nature Reviews Immunology, v.8, n.5, p.685-698, 2010.

PASSOS, K.E.; BERNARDI, J.R.; MENDES, K.G. Análise da composição nutricional da Cesta Básica brasileira. Ciência \& Saúde Coletiva, v.19, n.5, p.1623-1630, 2014.

PINKOSKY, S.L.; GROOT, P.H.E.; LALWANI, N.D.; STEINBERG, G.R. Targeting ATPCitrate lyase in hyperlipidemia and metabolic disorders. Trends in Molecular Medicine, v.23, n.11, p.1047-1063, 2017.

POF. Pesquisas de Orçamentos Familiares 2008-2009: Análise do Consumo Alimentar Pessoal no Brasil, 2011.

PROCON. Programa de Defesa do Consumidor - Prefeitura de Joinville. Pesquisa de Preços dos Produtos da Cesta Básica, 3p., 2016. 
PU, F.; CHEN, N.; XUE, S. Calcium intake, calcium homeostasis and health. Food Science and Human Wellness, v.5, n.1, p.8-16, 2016.

SALTIEL, F.; URZÚA, S. The effect of the minimum wage on Employment in Brazil. Development Bank of Latin America, n.22, p.1-33, 2017.

SALVA, S.M.C.; MERINO, G.A.; GRUPPI, A; ALVAREZ, S. Dietary supplementation with probiotics improve hematopoiesis in malnourished mice. PLoS One, v.7, n.2, p.31171, 2012.

SCHWINGSHACKL, G.; HOFFMANN, L. Comparasion off effects of long-term low-fat vs high-fat diet on blood lipid levels in overweight or obese patients: a systematic review and meta-analysis. Journal of The Academy of Nutrition and Dietetics, v.113, n.12, p.1640-1661, 2013.

SOMMER. Vitamin A deficiency and clinical disease: an historical overview. Journal of Nutrition, v.138, n.10, p.1835-1839, 2008.

UDAY, S.; HOGLER, W. Nutritional rickets and Osteomalacia in the Twenty-first Century: Revised Concepts, public health, and prevention strategies. Current Osteoporosis Reports, v.15, n.4, p.293-302, 2017.

VANNUCCI, L.; MASI, L.; GRONCHI, G.; FOSSI, C.; CAROSSINO, A.M.; BRANDI, M.L. Calcium intake, bone mineral density, and fragility fractures: evidence from an Italian outpatient population. Archive of Osteoporosis, v.12, n.1, 2017.

WEDNER, S.H.; ROSS, D.A. Vitamin A deficiency and Its Prevention. Reference Module in Biomedical Sciences - International Encyclopedia of Public Health (Second Edition), p.378382, 2017.

ZHOU, Y.; XUE, S.; YANG, J.J. Calciomics: integrative studies of $\mathrm{Ca}+2$-binding proteins and their interactomes in biological systems. Metallomics, v.5, n.1, p.29-42, 2013. 